\title{
Efecto del aceite esencial de Chrysactinia mexicana A. Gray sobre aislados clínicos de Candida glabrata
}

\author{
Effect of the essential oil of Chrysactinia mexicana A. Gray on clinical isolates of Candida glabrata
}

\begin{abstract}
Omar Medina-de la Cruza, Carlos Alberto Leal-Morales ${ }^{b}$, Thuluz Meza-Menchacac, Larissa Guillend, Bertha Irene Juárez-Florese, Verónica Gallegos-García*a

a Facultad de Enfermería y Nutrición, Universidad Autónoma de San Luis Potosí, San Luis Potosí, México. Av. Niño Artillero No. 130. Zona Universitaria. C.P. 78240.

b Departamento de Biología, División de Ciencias Naturales y Exactas, Campus Guanajuato, Universidad de Guanajuato, Guanajuato, México. Noria Alta S/N. Col. Noria Alta. C.P. 36050.

Laboratorio de Genómica Humana, Facultad de Medicina, Universidad Veracruzana, Xalapa, México. Calle Médicos y Odontólogos S/N. Col. Unidad del Bosque. C.P. 91010.

d Red de Manejo Biorracional de Plagas y Vectores, Instituto de Ecología, A.C. (INECOL), Clúster Científico y Tecnológico BioMimic, Xalapa, México. Carretera Antigua a Coatepec No. 351. Col. El Haya. C.P. 91070.

e Facultad de Ciencias Químicas, Universidad Autónoma de San Luis Potosí, San Luis Potosí, México. Av. Dr. Manuel Nava Martínez No. 6. Zona Universitaria. C.P. 78210.
\end{abstract}

\section{RESUMEN}

El objetivo fue evaluar el efecto del aceite esencial de Chrysactinia mexicana A. Gray en aislados clínicos de Candida glabrata. Se obtuvo aceite esencial por arrastre de vapor de las flores, hojas y ramas secundarias de C. mexicana. Se realizó cromatografía de gases y espectrometría de masas para conocer la composición del aceite. Se hicieron ensayos con aislados clínicos de C. glabrata y Saccharomyces cerevisiae (control), para determinar la Concentración Mínima Inhibitoria (CMI) del aceite. Los cultivos se incubaron en medio YPD líquido, $7 \mathrm{~h}$ en medio nuevo (fase logarítmica) o $3 \mathrm{~h}$ en medio recuperado de fase estacionaria (fase estacionaria) con el aceite y se realizaron ensayos de microdilución. Los principales tres compuestos del aceite fueron la piperitona (29.57\%), eucaliptol (26.86\%) y a-terpineol (14.65\%). Las CMls obtenidas fueron, a) fase estacionaria: S. cerevisiae (BY4741) 2.78 $\mathrm{mg} / \mathrm{mL}$ y C. glabrata (CBS138), (NY66) y (AN400) $6.50 \mathrm{mg} / \mathrm{mL}$ y (NY62) $9.29 \mathrm{mg} / \mathrm{mL}$; b) fase logarítmica: S. cerevisiae (BY4741) $3.71 \mathrm{mg} / \mathrm{mL}$ y C. glabrata (CBS138) $4.64 \mathrm{mg} / \mathrm{mL}$, (NY66) 6.5 $\mathrm{mg} / \mathrm{mL}$, (AN400) $8.36 \mathrm{mg} / \mathrm{mL}$, y (NY62) $18.5 \mathrm{mg} / \mathrm{mL}$. Este trabajo constituye el primer reporte del efecto fungicida del aceite esencial de $C$. mexicana sobre hongos patógenos del humano como C. glabrata.

Palabras clave: S. cerevisiae, Fungicida, Piperitona, Asteraceae, Microdilución

\section{ABSTRACT}

The objective of this work was to evaluate the effect of the Chrysactinia mexicana A. Gray essential oil, on C. glabrata clinical isolates. The essential oil was obtained by steam entrainment of flowers, leaves and secondary branches of $C$. mexicana. Gas chromatography and mass spectrometry were performed to determine the oil composition. Logarithmic and stationary phase microdilution assays were performed with Saccharomyces cerevisiae and clinical isolates of C. glabrata to determine the Minimum Inhibitory Concentration
(MIC) of the oil. The cultures were incubated in liquid YPD medium, for $7 \mathrm{~h}$ in fresh medium (logarithmic phase) or $3 \mathrm{~h}$ in recovering medium (stationary phase), with the oil and microdilution tests were performed. The three main oil compounds identified were piperitone (29.57\%), eucalyptol (26.86\%) and a-terpineol (14.65\%). The MICs obtained were, a) stationary phase: S. cerevisiae (BY4741) $2.78 \mathrm{mg} / \mathrm{mL}$ and C. glabrata (CBS138), (NY66) and (AN400) $6.50 \mathrm{mg} / \mathrm{mL}$ and (NY62) $9.29 \mathrm{mg} / \mathrm{mL}$; b) logarithmic phase: S. cerevisiae (BY4741) $3.71 \mathrm{mg} / \mathrm{mL}$ and C. glabrata (CBS138) $4.64 \mathrm{mg} / \mathrm{mL}$, (NY66) $6.5 \mathrm{mg} / \mathrm{mL}$, (AN400) $8.36 \mathrm{mg} / \mathrm{mL}$, and (NY62) $18.5 \mathrm{mg} /$ $\mathrm{mL}$. This work constitutes the first report of the fungicidal effect of the C. mexicana essential oil on human pathogenic fungi such as C. glabrata.

Keywords: S. cerevisiae, Fungicide, Piperitone, Asteraceae, Microdilution

\section{INTRODUCCIÓN}

Las infecciones fúngicas invasivas han aumentado desde la década de los 80 's en pacientes inmunosuprimidos por trasplante o portadores del virus de inmunodeficiencia humana, personas con tratamiento de quimioterapia, y aquellos que reciben terapias prolongadas con antibióticos de amplio espectro son vulnerables al desarrollo de este tipo de micosis (Santos et al., 2018).

Los microorganismos causantes de estas infecciones son las especies del género Candida, que forman parte de la microbiota y se localizan en la mucosa del tracto gastrointestinal, vagina e incluso la piel (Underhill y lliev, 2014). Al presentarse las condiciones que favorecen su desarrollo, pueden pasar de causar una infección mucocutánea a ocasionar una candidemia debido a su adaptabilidad a los diferentes microambientes del huésped (Eggimann et al., 2003; da Silva et al., 2016).

Se han identificado más de 200 especies del género Candida, aunque alrededor de 31 producen enfermedades

*Autor para correspondencia:Verónica Gallegos García Correo electrónico: veronica.gallegos@uaslp.mx

Recibido: 7 de abril de 2020

Aceptado: 11 de octubre de 2020 
en el humano, de éstas más del $90 \%$ de las infecciones son causadas por C. albicans, C. glabrata, C. parapsilosis, C. kruzei y C. tropicalis (Eggimann et al., 2003). C. albicans es la especie que se aísla con mayor frecuencia en pacientes, pero en los últimos años se incrementó el número de infecciones por Candida spp. (Magalhães et al., 2015).

En Islandia, Finlandia, Noruega, Dinamarca, Estados Unidos de América y España C. glabrata se aísla de enfermos con candidemia en segundo lugar de frecuencia (Guinea, 2014). Un reporte en 2015 de la Secretaria de Salud sobre las Infecciones Asociadas a la Atención en la Salud (IAAS) de 378 unidades notificantes, reportó a C. glabrata dentro de los 20 principales agentes etiológicos de IAAS con 203 casos (SSA, 2015). Aunque, en México se carece de estudios epidemiológicos recientes y detallados que indiquen la prevalencia de C. glabrata en pacientes con candidemia (Pineda-Díaz et al., 2017).

C. glabrata es un patógeno emergente que tiene un mayor nivel de resistencia a los medicamentos para su tratamiento a base de azoles y equinocandinas en comparación con C. albicans (Horn et al., 2009). Las infecciones por C. glabrata se asocian con aumento en los días de estancia hospitalaria y por consecuencia a un mayor costo por su atención (Moran et al., 2010).

Debido a la resistencia que tiene C. glabrata a los medicamentos, se ha propuesto el uso de plantas medicinales que se han utilizado para diferentes padecimientos (Mahilrajan et al., 2014). Entre los países que utilizan la medicina tradicional se encuentran China, Brasil, Cuba, India, Jordania y México, por la riqueza y diversidad de su flora la cual puede tener diversas aplicaciones biológicas (Bakkali et al., 2008).

Existen reportes en plantas de la familia Asteraceae sobre la actividad biológica de los aceites esenciales extraídos de éstas. Un ejemplo es Chrysactinia mexicana A. Gray a quien se le han atribuido efectos como: afrodisiaco, antiespasmódico, antidiarreico, diurético, tónico, antipirético, antiinflamatorio y antifúngico (Cárdenas-Ortega et al., 2005; García-López et al., 2017). Sobre la actividad antifúngica del aceite esencial de C. mexicana fue utilizado por Cárdenas-Ortega et al. (2005) sobre Aspergillus flavus, microorganismo fitopatógeno que afecta al maíz. También existen reportes de extractos acuosos y metanólicos de $C$. mexicana sobre aislados clínicos de Escherichia coli, Shigella, Salmonella, Mycobacterium tuberculosis (Alanis et al., 2005: García-López et al., 2017).

Sin embargo, no hay reportes del aceite esencial de $C$. mexicana sobre hongos patógenos como C. glabrata, además es poco lo que se conoce sobre su posible mecanismo de acción, por lo tanto, es de interés evaluar el efecto del aceite esencial de esta planta sobre aislados clínicos de C. glabrata y de un hongo no patógeno de humanos como Saccharomyces cerevisiae, que es filogenéticamente más relacionado a $C$. glabrata (Castaño et al., 2006).

\section{MATERIALES Y MÉTODOS Microorganismos utilizados}

Las cepas empleadas en este estudio se presentan en la Tabla1. La identificación molecular de los aislados clínicos de C. glabrata se realizó por PCR de acuerdo a lo reportado en 2015 (Castaño et al., 2015).

\section{Medios de cultivo}

Las células se cultivaron en medio de levadura estándar YPD (del inglés Yeast extract-Peptone-Dextrose). El medio líquido contenía $1 \%$ de extracto de levadura (BD Bioxon), $2 \%$ peptona (BD Bioxon) y $2 \%$ de glucosa (Fermont), el medio sólido se preparó al adicionar 2\% agar (BD Bioxon).

\section{Recolección de C. mexicana}

C. mexicana (Asteraceae) en etapa de floración se recolectó en el mes de Julio del año 2016 en el camino a Guadalcázar municipio de San Luis Potosí en las coordenadas $22^{\circ} 36^{\prime} 30.2^{\prime \prime} \mathrm{N} 100^{\circ} 26^{\prime} 50.2^{\prime \prime} \mathrm{O}$. El material vegetal fue autenticado con el código SLPM37571 y depositado en el herbario del Instituto de Zonas Desérticas de la Universidad Autónoma de San Luis Potosí.

\section{Obtención del aceite esencial de C. mexicana}

El material vegetal se secó a temperatura ambiente por $72 \mathrm{~h}$, se separaron las flores, las hojas y las ramas secundarias; se trituraron y se realizó la molienda en seco para obtener una partícula de $1.75 \mathrm{~mm}$ para la homogenización de la muestra. El aceite esencial se extrajo por la técnica de destilación por arrastre de vapor, la técnica para separar el aceite esencial del agua floral fue la separación por densidades con un embudo de decantación (Cárdenas-Ortega et al., 2005).

\section{Cromatografía de gases y espectrometría de masas}

Para el análisis de la composición química del aceite esencial de C. mexicana extraídos de una mezcla de flores, las hojas y las ramas secundarias por destilación, se utilizó un cromatógrafo de gases (Agilent 7890B) equipado con una columna ZB-5MSi ( $30 \mathrm{~m}$ X $0.25 \mathrm{~mm}$ ID X $0.25 \mu \mathrm{m}$ ) y acoplado

Tabla 1. Cepas de levaduras empleadas en este estudio. Table 1. Yeast strains used in this study.

\begin{tabular}{|c|c|c|c|}
\hline Cepa & Microorganismo & Origen/Descripción & Referencia \\
\hline BY4741 & S. cerevisiae & MATa his $3 \Delta 1$ leu $2 \Delta 0$ met $15 \Delta 0$ ura $3 \Delta 0$ & (Baker-Brachmann et al., 1998) \\
\hline CBS138 & C. glabrata & ATCC2001 & (Dujon et al., 2004) \\
\hline AN400 & C. glabrata & Aislado clínico de hemocultivo & Este estudio \\
\hline NY62 & C. glabrata & Aislado clínico de urocultivo & Este estudio \\
\hline NY66 & C. glabrata & Aislado clínico de exudado vaginal & Este estudio \\
\hline
\end{tabular}


a un analizador de masas TQ 7000D Agilent Technologies. Como gas de arrastre se empleó helio a un flujo constante de $1.2 \mathrm{~mL} / \mathrm{min}$. Se inyectó $1 \mu \mathrm{L}$ de una dilución de la muestra $(1 \mu \mathrm{L}$ de muestra en $199 \mu \mathrm{L}$ diclorometano grado HPLC, Sigma-Aldrich) en modo "split" (16.7 mL/min) con el inyector a $250^{\circ} \mathrm{C}$.

Las condiciones cromatográficas iniciaron con una temperatura de $50^{\circ} \mathrm{C}$ por $2 \mathrm{~min}$, y se incrementó la temperatura $15^{\circ} \mathrm{C}$ por min hasta alcanzar $280^{\circ} \mathrm{C}$, manteniendo ésta temperatura por $10 \mathrm{~min}$. La temperatura de la interfase y fuente del EM fueron $250^{\circ} \mathrm{C}$ y $230^{\circ} \mathrm{C}$, respectivamente. La detección de analitos se hizo en modo de barrido total del espectro (scan) de 30 a $500-\mathrm{m} / \mathrm{z}$ con un detector de IE. La identificación de los analitos fue realizada con la librería NIST (National Institute of Standards and Technology) versión 2.0D, NIST/EPA/NIH (NIST05) y con el uso de estándares sintéticos.

\section{Determinación de la concentración mínima inhibitoria en la fase logarítmica o estacionaria de crecimiento del microorganismo con aceite esencial de $C$. mexicana}

La CMI se determinó como la concentración más baja del aceite esencial de $C$. mexicana requerida para la inhibición completa del crecimiento de C. glabrata (Zore et al., 2011). A partir de un precultivo de C. glabrata de $48 \mathrm{~h}$, se inocularon tubos falcon con $10 \mathrm{~mL}$ de medio YPD a una OD600 ${ }_{\mathrm{nm}} 0.5$. El control contenía o no dimetilsulfoxido (DMSO) al $1.25 \%$ del volumen final (D8418 Sigma-Aldrich) del diluyente del aceite esencial, y se adicionaron las diferentes concentraciones del aceite esencial de $C$. mexicana que fueron desde $0.92 \mathrm{mg} / \mathrm{mL}$ hasta $18.5 \mathrm{mg} / \mathrm{mL}\left(\rho=0.9290 \mathrm{~g} / \mathrm{cm}^{3}\right)$ dependiendo de la cepa.

Los cultivos se incubaron a $28^{\circ} \mathrm{C}$ con agitación continua a 120 rpm durante $7 \mathrm{~h}$ en medio nuevo (fase logarítmica de crecimiento) o durante $3 \mathrm{~h}$ en medio recuperado de fase estacionaria (fase estacionaria de crecimiento), transcurrido el tiempo de incubación se determinó la densidad óptica y se ajustó a $\mathrm{OD}_{600 \mathrm{~nm}} 0.5$ en $1 \mathrm{~mL}$ de agua destilada estéril, posteriormente se lavaron las células y se centrifugaron a 13,000 rpm durante $30 \mathrm{~s}$ y se realizaron ensayos de microdilución (Cuéllar-Cruz et al., 2008) en placas de 96 pocillos y una alícuota de $180 \mu \mathrm{L}$ se colocaron en cajas de petri con medio YPD agar durante $48 \mathrm{~h}$.

\section{Conteo de unidades formadoras de colonias}

A partir del crecimiento observado de S. cerevisiae y C. glabrata en los ensayos de microdilución sobre YPD sólido fueron seleccionadas las diluciones donde el número de colonias eran contables y posteriormente de cada una de ellas se espatularon $25 \mu \mathrm{L}$ sobre el medio YPD sólido. Se incubaron durante $48 \mathrm{~h}$ a $28^{\circ} \mathrm{C}$ para posteriormente realizar el conteo de las Unidades Formadoras de Colonias (UFC).

\section{Análisis estadísticos}

Se utilizó el programa estadístico GraphPad Prism V.5. Para la comparación de los diferentes tratamientos en cada cepa se hizo la prueba estadística de Shapiro-Wilk para después realizar la prueba ANOVA de una vía considerando el conteo de UFC como la variable de respuesta. Para evaluar la diferencia entre las medias de los grupos (IC=95 \% y $p=0.05)$ se utilizaron análisis post-hoc con la prueba de Tukey los cuales se presentan en las respectivas gráficas.

\section{Predicción de los espectros de actividad para las sustan- cias}

Se realizó un análisis in silico para la predicción de los espectros de actividad para las sustancias con los principales compuestos del aceite esencial para conocer el posible mecanismo de acción, con el software gratuitamente http:// www.way2drug.com/RA. La estructura química se cargó con la herramienta de Marvin. Los resultados de la predicción muestran la estructura con los átomos numerados y una tabla con el espectro probable de la reacción de biotransformación. Este espectro se calcula mediante el software PASS, basado en el análisis SAR. La precisión promedio de la predicción en validación cruzada de LOO (IAP) es 0.86 mientras el valor este más cercano a 1 más posibilidad tiene de actuar de esa manera.

\section{RESULTADOS}

\section{Composición del aceite esencial de C. mexicana}

La composición química del aceite esencial de C. mexicana se muestra en la Tabla 2, en donde se identificaron 26 compuestos. El aceite se caracterizó por la presencia mayoritaria de cinco compuestos: piperitona (29.58\%), eucaliptol (26.86\%), a-terpineol (14.65\%), delta-3-careno (12.37\%) y linalool (3.56\%).

Efecto del aceite esencial de $C$. mexicana sobre S. cerevisiae y C. glabrata en la fase logarítmica de su crecimiento

Para la cepa de S. cerevisiae la CMI fue de $3.71 \mathrm{mg} / \mathrm{mL}$, esta concentración es menor respecto a la utilizada en las cepas de C. glabrata. Para las cepas CBS138, NY66, AN400 y NY62 la CMI fue de $4.64 \mathrm{mg} / \mathrm{mL}, 6.5 \mathrm{mg} / \mathrm{mL}, 8.36 \mathrm{mg} / \mathrm{mL}$ y $18.5 \mathrm{mg} / \mathrm{mL}$ respectivamente (Figura 1 ).

Con base en el conteo de UFC, se realizó la prueba estadística de ANOVA para determinar la diferencia de las medias de las cepas utilizadas con las distintas concentraciones de aceite esencial en la fase logarítmica de crecimiento. Se observa que en todas las cepas utilizadas hay una diferencia estadísticamente significativa $(p=0.001)$ entre los controles con la CMI determinada por el crecimiento sobre agar sólido. En los controles sin y con diluyente no hay diferencia estadística (Figura 2).

Efecto del aceite esencial de C. mexicana sobre S. cerevisiae y C. glabrata en la fase estacionaria de su crecimiento

En la fase estacionaria de crecimiento se encontró que en S. cerevisiae la CMI fue $2.78 \mathrm{mg} / \mathrm{mL}$, para la cepa CBS138 la CMI fue de $6.50 \mathrm{mg} / \mathrm{mL}$ esta concentración fue la misma que para la cepa NY66 y la cepa AN400, sin embargo, para el aislado clínico NY62 la CMI fue mayor con $9.29 \mathrm{mg} / \mathrm{mL}$ (Figura 3).

Los análisis estadísticos indican que entre el control sin diluyente y el que tenía DMSO al $1.25 \%$ no hay una diferencia estadísticamente significativa, por lo cual, el diluyente 
Tabla 2. Compuestos identificados mediante CG-EM en el aceite esencial de C. mexicana.

Table 2. Compounds identified by GC-MS in the C. mexicana essential oil.

\begin{tabular}{|c|c|c|c|}
\hline No. & Compuestos & $\begin{array}{l}\text { Tiempo de } \\
\text { Retención } \\
\text { (min) }\end{array}$ & $\begin{array}{l}\text { Abundancia } \\
\text { Área del pico (\%) }\end{array}$ \\
\hline 1 & alfa-pinene* & 4.980 & 0.058 \\
\hline 2 & alfa-felandreno* & 5.257 & 1.393 \\
\hline 3 & Camfeno & 5.438 & 0.820 \\
\hline 4 & Benzaldehído & 5.589 & 0.025 \\
\hline 5 & Sabineno & 5.745 & 0.528 \\
\hline 6 & beta-pineno & 5.820 & 0.473 \\
\hline 7 & Mirceno & 5.949 & 0.474 \\
\hline 8 & alfa-terpineno & 6.301 & 0.678 \\
\hline 9 & p-cimeno* & 6.379 & 2.349 \\
\hline 10 & Eucaliptol* & 6.497 & 26.863 \\
\hline 11 & Delta-3-careno & 6.611 & 12.368 \\
\hline 12 & gamma-terpineno & 6.762 & 0.469 \\
\hline 13 & Terpinoleno & 7.138 & 0.198 \\
\hline 14 & Linalool* & 7.210 & 3.564 \\
\hline 15 & Fenil acetato de metilo & 8.072 & 0.209 \\
\hline 16 & Terpinen-4-ol & 8.117 & 0.229 \\
\hline 17 & Alfa-terpineol* & 8.236 & 14.654 \\
\hline 18 & Cis-3-hexenil isovalerato & 8.639 & 0.064 \\
\hline 19 & Piperitona* & 8.925 & 29.576 \\
\hline 20 & Indole & 9.255 & 0.243 \\
\hline 21 & Metil antranilato & 9.641 & 0.162 \\
\hline 22 & Eugenol & 9.849 & 0.538 \\
\hline 23 & Cis-jasmone & 10.142 & 0.412 \\
\hline 24 & beta-cariofileno & 10.448 & 1.153 \\
\hline 25 & Valenceno & 10.838 & 1.190 \\
\hline 26 & Acetato de guayilo & 11.646 & 1.629 \\
\hline
\end{tabular}

*Compuestos verificados con estándares

no afecta el crecimiento de las células. Un dato importante es que en todas las cepas hubo diferencia entre los controles y las concentraciones de aceite esencial de C. mexicana probados. Aunque para la cepa de S. cerevisiae, el aislado NY66 y el aislado NY62, la segunda concentración utilizada, ya no muestra diferencias estadísticas respecto a la concentración anterior probada (Figura 4).

\section{Predicción de los espectros de actividad para las sustan- cias}

Con base a la predicción de los espectros, se propone el siguiente mecanismo de acción con los compuestos mayoritarios (Figura 5). La piperitona fue el compuesto en mayor porcentaje en el aceite esencial que se encontró y que inhibe la actividad de la fosfatasa con un valor (Potencial Inhibidor)
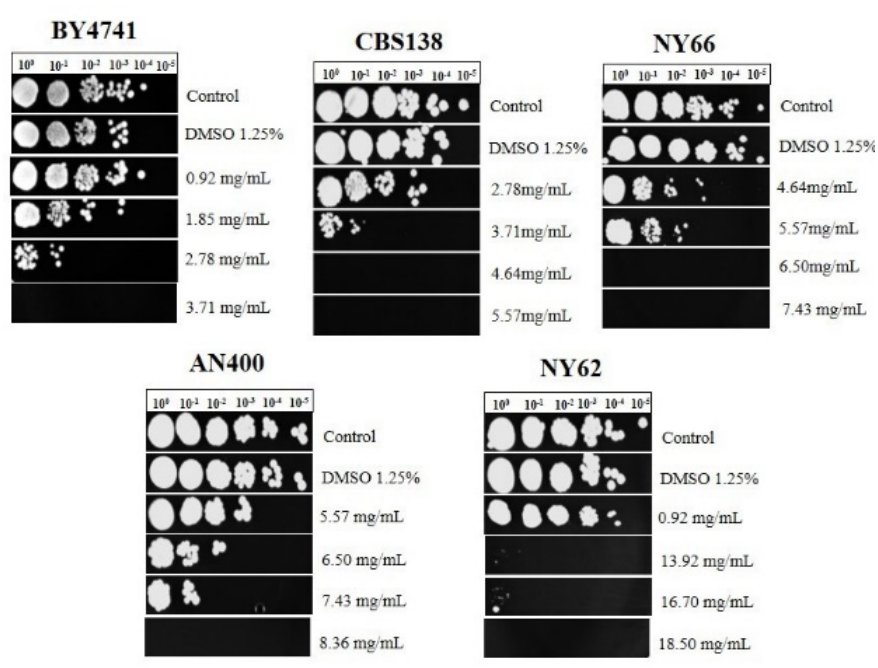

Figura 1. Efecto del aceite esencial de C. mexicana sobre S. cerevisiae y $C$. glabrata en la fase logarítmica de crecimiento. Ensayo de microdilución $\left(10^{\circ}\right.$ a la 10-5). BY4741: cepa de S. cerevisiae. Cepas de C. glabrata; CBS138: Cepa ATCC. NY66: aislado clínico de exudado vaginal. AN400: aislado clínico de hemocultivo. NY62: aislado clínico de urocultivo. Líneas: crecimiento de la cepa sin diluyente y sin aceite esencial (control), con diluyente (DMSO 1.25 $\%$ ) y sin aceite esencial [controles]; con las diferentes concentraciones del aceite esencial $(\mathrm{mg} / \mathrm{mL})$. Incubación de $48 \mathrm{~h}$ sobre medio YPD agar a $28^{\circ} \mathrm{C}$. Los ensayos se realizaron 5 veces.

Figure 1. Effect of C. mexicana essential oil on S. cerevisiae and C. glabrata growth at the logarithmic phase.

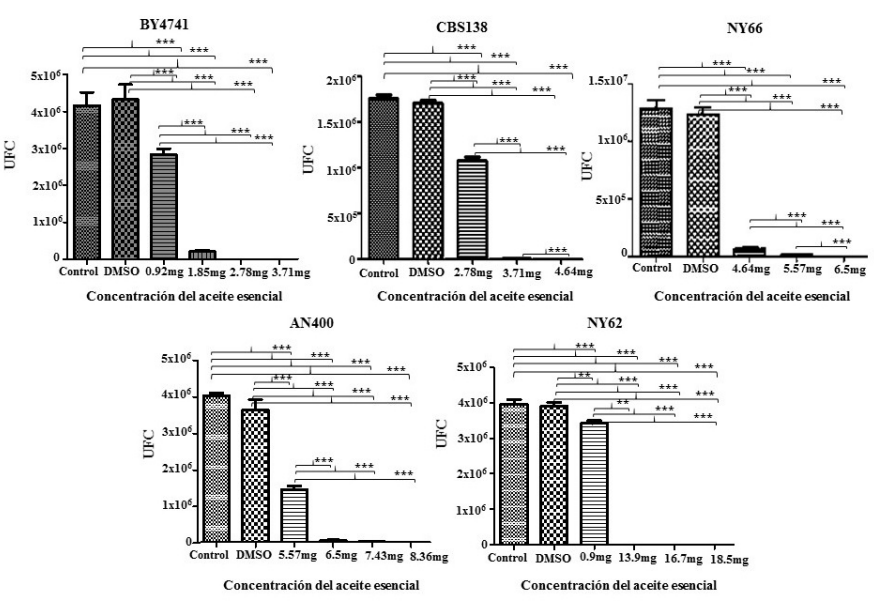

Figura 2. Análisis de ANOVA del efecto del aceite esencial de C. mexicana sobre S. cerevisiae y C. glabrata en fase logarítmica de crecimiento. BY4741: cepa de S. cerevisiae. Cepas de C. glabrata; CBS138: Cepa ATCC. NY66: aislado clínico de exudado vaginal. AN400: aislado clínico de hemocultivo. NY62: aislado clínico de urocultivo. En el eje de las X se observa las diferentes concentraciones del aceite esencial probadas, mientras que en el eje de las Y las Unidades Formadoras de Colonias (UFC). Los ensayos se realizaron 5 veces. Los corchetes con asteriscos arriba de las barras se muestran solo en las comparaciones entre tratamientos que tuvieron diferencias significativas $\left({ }^{* *} p=0.01\right)$ o altamente significativa $\left({ }^{* * *} p=0.001\right)$.

Figure 2. ANOVA analysis of the effect of $C$. mexicana essential oil on $S$. cerevisiae and C. glabrata growth at the logarithmic phase. 


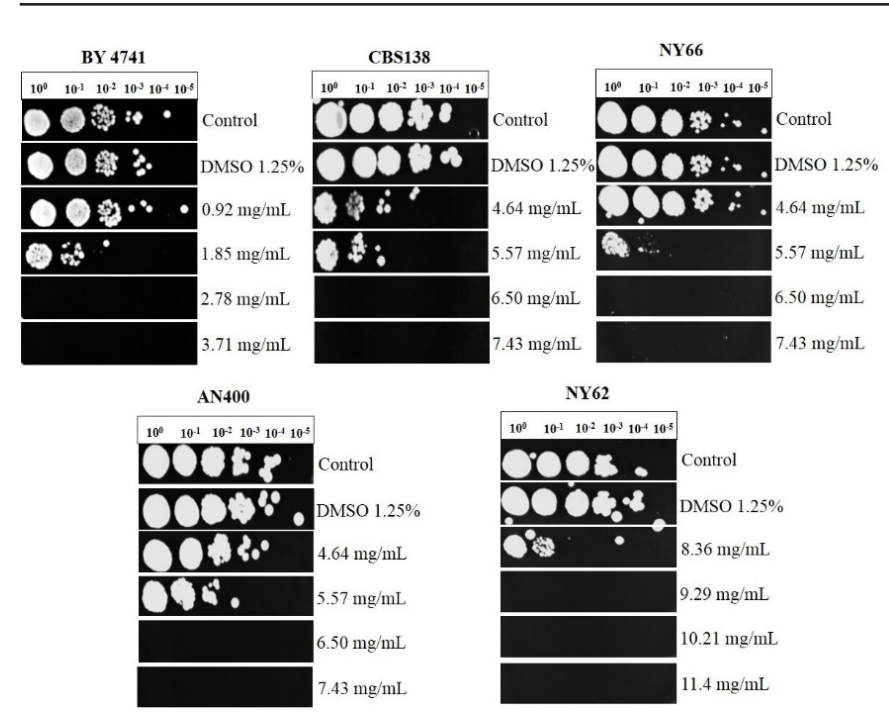

Figura 3. Efecto del aceite esencial de $C$. mexicana sobre $S$. cerevisiae y $C$. glabrata en fase estacionaria de crecimiento. Ensayo de microdilución $\left(10^{\circ}\right.$ a la 10-5). BY4741: cepa de S. cerevisiae. Cepas de C. glabrata; CBS138: Cepa ATCC. NY66: aislado clínico de exudado vaginal. AN400: aislado clínico de hemocultivo. NY62: aislado clínico de urocultivo. Líneas: crecimiento de la cepa sin diluyente y sin aceite esencial (control), con diluyente (DMSO 1.25 $\%$ ) y $\sin$ aceite esencial [controles]; con las diferentes concentraciones del aceite esencial $(\mathrm{mg} / \mathrm{mL})$. Incubación de $48 \mathrm{~h}$ sobre medio YPD agar a $28^{\circ} \mathrm{C}$. Los ensayos se realizaron 5 veces.

Figure 3. Effect of C. mexicana essential oil on S. cerevisiae and C. glabrata growth at the stationary phase.

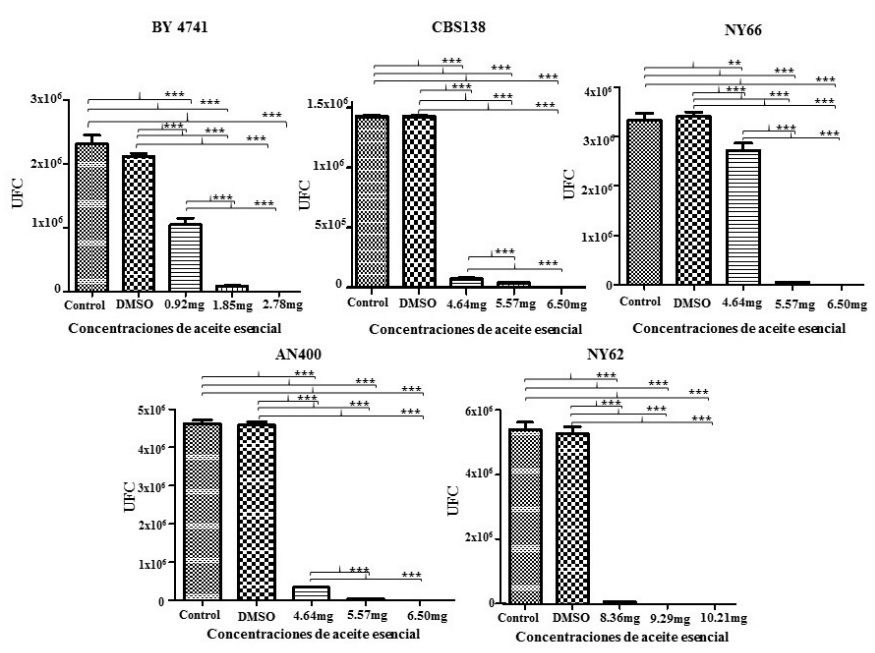

Figura 4. Análisis de ANOVA del efecto del aceite esencial de C. mexicana sobre S. cerevisiae y C. glabrata en fase estacionaria de crecimiento. BY4741: cepa de S. cerevisiae. Cepas de C. glabrata; CBS138: Cepa ATCC. NY66: aislado clínico de exudado vaginal. AN400: aislado clínico de hemocultivo. NY62: aislado clínico de urocultivo. En el eje de las X se observa las diferentes concentraciones del aceite esencial probadas, mientras que en el eje de las $Y$ las Unidades Formadoras de Colonias (UFC). Los ensayos se realizaron 5 veces. Los corchetes con asteriscos arriba de las barras se muestran solo en las comparaciones entre tratamientos que tuvieron diferencias significativas $\left({ }^{* *} p=0.01\right)$ o altamente significativa $\left({ }^{* * *} p=0.001\right)$.

Figure 4. ANOVA analysis of the effect of $C$. mexicana essential oil on $S$. cerevisiae and C. glabrata in the stationary growth phase.

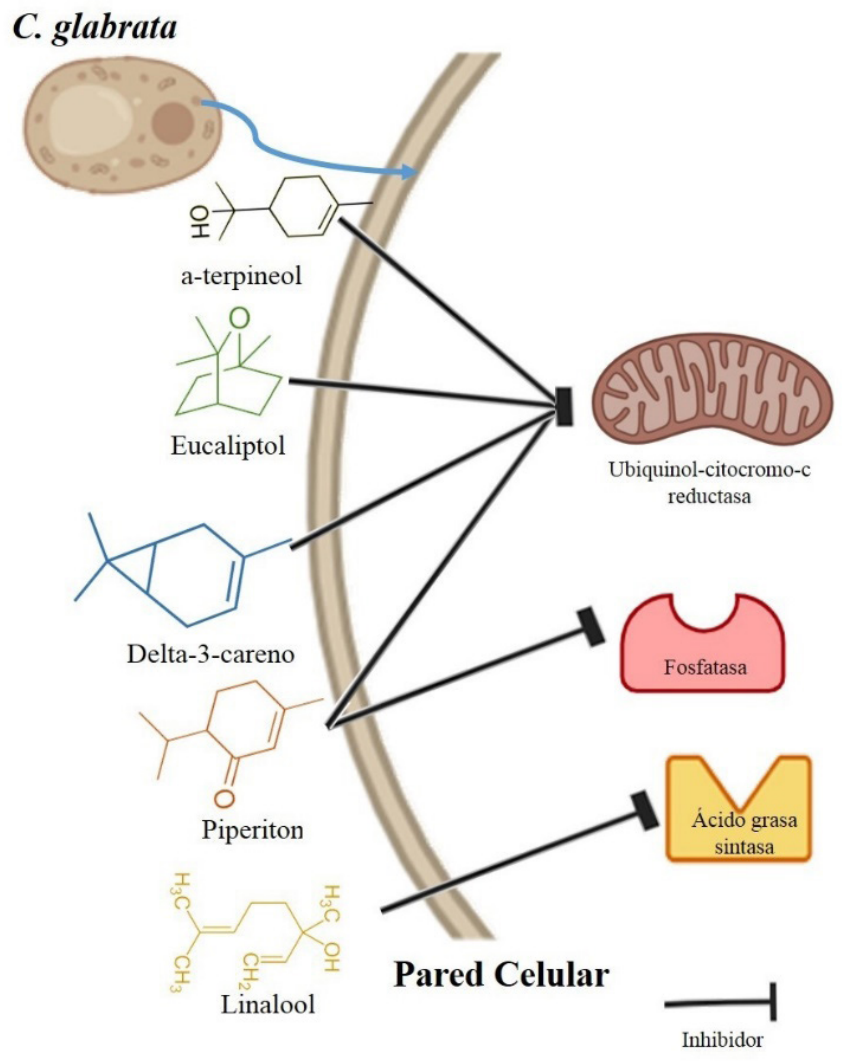

Figura 5. Mecanismo de acción propuesto de los principales compuestos del aceite esencial de $C$. mexicana. Se muestran los principales componentes piperitona, eucaliptol, linalool, delta-3-careno, a-terpineol y su posible efecto sobre las células de C. glabrata al pasar la pared celular. Como inhibidor de la enzima acido grasa sintasa se encuentra el linalool. Como inhibidor de la enzima fosfatasa el compuesto piperitona y el delta-3-careno. Como inhibidor de la Ubiquinol-citocromo-c- reductasa se encuentran la piperitona, eucaliptol, delta-3-careno y el a-terpineol. Elaboración propia con el programa biorender.

Figure 5. Proposed mechanism of action of the main essential oil compounds of $C$. Mexicana.

$\mathrm{Pl}=0.783$, y que también tiene un efecto como probable inhibidor de la ubiquinol-citocromo-c reductasa con $\mathrm{Pl}=0.873$, al igual que el eucaliptol con un valor $\mathrm{Pl}=0.760$.

El linalool tiene un valor de $\mathrm{Pl}=0.868$ como inhibidor de la ácido grasa sintasa, el delta-3-careno también inhibe la ubiquinol-citocromo-c reductasa con $\mathrm{Pl}=0.800$ al igual que el compuesto $a$-terpineol con un $\mathrm{Pl}=0.775$.

\section{DISCUSIÓN}

Se ha demostrado que aislados clínicos de C. glabrata presentan resistencia moderada a los azoles y equinocandinas, estos pueden volverse más resistentes después del uso de los fármacos (Jiang et al., 2013). Aunque la información del mecanismo de acción de los aceites esenciales en hongos es limitada en comparación con los reportes en bacterias, se ha señalado que la actividad de los aceites esenciales en levaduras se debe al daño ocasionado en alguno de los sistemas enzimáticos involucrados en la producción de 
energía y síntesis de componentes de la estructura celular (Hyldgaard et al., 2012; Nazzaro et al., 2013).

Las concentraciones del aceite esencial de C. mexicana para inhibir el crecimiento de S. cerevisiae y C. glabrata son mayores que las reportadas por Cárdenas-Ortega et al. (2005) para Aspergillus flavus, una posible explicación es que la metodología utilizada es diferente ya que en ese estudio utilizaron el método de difusión en agar. Además, se ha planteado que los mamíferos como el ser humano son altamente resistentes a enfermedades fúngicas invasivas como la provocada por C. glabrata de allí que los microorganismos fúngicos patógenos del ser humano sean más resistentes a los medicamentos que los fitopatógenos ya que deben cumplir cuatro criterios: 1) poder crecer a altas temperaturas $\left(>37^{\circ} \mathrm{C}\right)$; 2) penetrar las barreras de los tejidos del huésped; 3 ) digerir y absorber componentes de los tejidos humanos y la más importante 4) evadir y resistir el sistema inmune humano (Köler et al., 2015). Es importante mencionar que $S$. cerevisiae es una levadura que no ocasiona enfermedades en el ser humano, sin embargo, es más cercana filogenéticamente a C. glabrata que a C. albicans.

La CMI de aceite esencial de $C$. mexicana en la cepa CBS138 durante la fase estacionaria fue más elevada que en fase logarítmica, lo anterior coincide con lo reportado por Jakubowski et al. (2000) quienes determinaron que las bacterias y hongos en la fase estacionaria son más resistentes al estrés oxidativo. Lo anterior se debe a que utilizan diferentes mecanismos enzimáticos en comparación con la fase logarítmica, ya que una vez que la célula entra en fase estacionaria deja de dividirse y la actividad metabólica se reduce, además de que activa distintas rutas metabólicas mediante genes que codifican para enzimas antioxidantes como catalasas, super-oxido dismutasa y síntesis de glutatión (Jakubowski et al., 2000).

La concentración del aceite esencial utilizada en los aislados clínicos AN400, NY62 y NY66 fue diferente con relación a las cepas de referencia (laboratorio), ya que las CMls en los aislados fue mayor en fase logarítmica que en fase estacionaria de su crecimiento, una posible explicación es que al utilizar cepas de Candida adaptadas al laboratorio estas se han subcultivado en varias ocasiones e incluso por décadas en algunos casos desde su primer aislamiento, por lo tanto, pueden ser un no representante adecuado para las cepas clínicamente relevantes en la actualidad esto se ha observado en la formación de biofilms (Alnuaimi et al., 2013). Se ha demostrado también que la microevolución de los hongos como C. glabrata puede ocurrir bajo condiciones de crecimiento in vitro e in vivo y de esta manera afectar los principales factores de virulencia (Franzot et al., 1998; Brunke et al., 2014).

Otra posible respuesta es que los aislados clínicos de C. glabrata muestran cariotipos variables porque su genoma se reorganiza con frecuencia y se considera que éstos reordenamientos son el principal mecanismo de adaptación de este microorganismo (Ahmad et al., 2014). Las aneuploidías en estos aislados clínicos sugieren variaciones en la organi- zación de los cromosomas y el número de copias de éstos son un mecanismo utilizado para generar rápidamente una respuesta a condiciones estresantes de crecimiento como la exposición a los antifúngicos, en este caso al aceite esencial (Selmecki et al., 2010).

Entre aislados clínicos existe variación en el tamaño de los cromosomas, sin dejar de lado la capacidad que posee C. glabrata para formar nuevos cromosomas cuyo origen son las duplicaciones segmentarias; éstos nuevos cromosomas llevan genes duplicados en su mayoría de la familia de transportadores $A B C$ que desempeñan un papel importante en la resistencia a múltiples fármacos (Poláková et al., 2009). Esto pudiera ser una posible explicación de por qué los aislados clínicos probados en este experimento son susceptibles a diferentes concentraciones del aceite esencial, aunque se deben realizar más investigaciones para poder determinarlo.

López-Fuentes et al. (2018) mencionan que la variabilidad del genoma de C. glabrata le confiere un patrón único de expresión de proteínas que se encuentran asociadas a la pared celular. Aislados clínicos de un paciente a menudo muestran 2 o 3 cariotipos diferentes y durante la infección el patrón cromosómico puede sufrir cambios en varios días. En C. glabrata las duplicaciones segmentarias, los reordenamientos cromosómicos y los cromosomas adicionales ocurren y persisten con alta frecuencia, así la elevada dinámica cromosómica no es compatible con el estilo de vida sexual y la meiosis, pero beneficia la adaptación a las condiciones ambientales cambiantes.

Algunas propuestas del mecanismo de acción de aceites esenciales son: 1) El sinergismo que producen todas las moléculas de los aceites, produce el efecto fungicida, aunque algunos investigadores como lpek et al. (2005) debaten que esto solo refleja el efecto de las principales moléculas que lo componen; 2) otro grupo de investigadores como Santana-Rios et al. (2001) y Hoet et al. (2006) sugieren que la actividad de los componentes mayoritarios está modulada por moléculas que se encuentran en un menor porcentaje y que es posible que varios componentes del aceite esencial le otorguen diferentes funciones: como el olor, la densidad, la textura, el color y sobre todo la capacidad de penetración celular y la forma en que se distribuye intracelularmente. Por lo tanto, habría que probar los compuestos por separado para poder confirmar alguna de las hipótesis.

Al realizar la predicción de los espectros de actividad para el eucaliptol, piperitona, delta-3-careno y el a-terpineol, se encontró que funcionan como posibles inhibidores de la ubiquinol-citocromo-c reductasa, la alteración de este gran complejo altera el estado redox de las células. Hoehamer et al. (2010) observaron que para C. albicans en interacción con agentes antifúngicos como azoles, polienos y equinocandinas muestran un cambio en el proteoma, por su interacción con éstos compuestos algunas proteínas se ven alteradas como la ubiquinona-citocromo-C-reductasa y comprometen el adecuado funcionamiento de las células.

La piperitona se encontró que actúa como un inhibidor de la enzima fosfatasa, la vía de transducción del fosfato 
en C. glabrata es similar que la de S. cerevisiae, esta ruta es esencial para sobrepasar la inanición (Orkwis et al., 2010). El linalool funciona como un posible inhibidor de la ácido grasa sintasa, la cual es muy importante porque está implicada en la síntesis de ácidos grasos de la pared celular. La cantidad de ácidos grasos sintetizados varía de acuerdo a las condiciones de crecimiento del microorganismo, además, en los hongos la síntesis de novo de ácidos grasos sucede en el citosol o en las mitocondrias (Roche et al., 2013).

Freisleben y Jäger proponen que el efecto antifúngico de los aceites esenciales puede ser por que alteran la estructura y función de la pared celular, así como las membranas de algunos organelos y la inhibición de proteínas o material nuclear (Freisleben y Jäger, 2014). Por otro lado, se ha demostrado que algunos aceites esenciales como el del árbol de té, Litsea cubeba y Anethum graveolens, actúan sobre C. albicans al alterar la permeabilidad y la fluidez de la pared celular, de esta manera se comprometen funciones asociadas a esta como la inhibición de ergosterol; esto los convierte en un posible potencial tratamiento. También pueden alterar el ciclo Krebs y la inhibición de la síntesis del ATP en las mitocondrias (Nazzaro et al., 2013).

\section{CONCLUSIONES}

El aceite esencial de $C$. mexicana es un posible potencial antifúngico, sin embargo, se debe ampliar la investigación para poder determinar la toxicidad en células del ser humano y de esta manera poder evaluar su uso de manera local o sistémica.

\section{AGRADECIMIENTOS}

Agradecemos amablemente a la Dra. Irene Castaño Navarro y al Dr. Alejandro De las Peñas Nava del Instituto Potosino de Ciencia y Tecnología por su apoyo en la donación de las distintas cepas de C. glabrata utilizadas en esta investigación. L.G. agradece el apoyo técnico de Mónica Navarro.

\section{CONFLICTO DE INTERES}

Los autores declaran no tener conflictos de intereses con respecto a los datos de esta publicación.

\section{FUENTE DE FINANCIACIÓN}

La presente investigación no ha recibido ninguna beca específica de agencias de los sectores público, comercial, o sin ánimo de lucro.

\section{REFERENCIAS BIBLIOGRAFÍCAS}

Alanis, A.D., Calzada, F., Cervantes, J.A., Torres, J.A., Torres, J. y Ceballos, G.M. 2005. Antibacterial properties of some plants used in Mexican traditional medicine for the treatment of gastrointestinal disorders. Journal of Ethnopharmacology. 100(1-2): 153-157.

Alnuaimi, A.D., O’Brien-Simpson, N.M., Reynolds, E.C. y McCullough, M.J. 2013. Clinical isolates and laboratory reference Candida species and strains have varing abilities to form biofilms. FEMS Yeast Research. 13(7): 689-699.
Ahmad, K.M., Kokošar, J., Guo, X., Gu, Z., Ishchuk, O.P. y Piškur, J. 2014. Genome structure and dynamics of the yeast pathogen Candida glabrata. FEMS Yeast Research. 14(4): 529-535.

Bakkali, F., Averbeck, S. y Averbeck, D., Idaomar, M. 2008. Biological effects of essential oils-a review. Food and Chemical Toxicology. 46(2): 446-475.

Baker-Brachmann, C., Davies, A., Cost, G.J., Caputo, E., Li, J., Hieter, P., et al. 1998. Designer deletion strains derived from Saccharomyces cerevisiae S288C: a useful set of strains and plasmids for PCR-mediated gene disruption and other applications. Yeast. 14(2): 115-132.

Brunke, S., Seider, K., Fischer, D., Jacobensen, I.D., Kasper, L., Jablonowski, N., et al. 2014. One small step for a yeastmicroevolution within macrophages renders Candida glabrata hypervirulent due to a single point mutation. Plos Pathogens. 10(10): e1004478.

Cárdenas-Ortega, N.C., Zavala-Sánchez, M.A., Aguirre-Rivera, J.R., Pérez-González, C. y Pérez-Gutiérrez, S. 2005. Chemical composition and antifungal activity of essential oil of Chrysactinia mexicana Gray. Journal of Agricultural and Food Chemistry. 53(11): 4347-4349.

Castaño, I.B., Cormack, B. y De Las Peñas, A. 2006. Virulencia del hongo patógeno oportunista Candida glabrata. Revista Latinoamericana de Microbiología. 48(2): 66-69.

Castaño, I.B., Cuéllar-Cruz, M. y De Las Peñas, A. 2015. Método in vitro para la detección de de Candida glabrata, kit de diagnóstico y uso de los mismos. México. EP2410052B1.

Cuéllar-Cruz, M., Briones-Martin-del-Campo, M., Cañas-Villamar, I., Montalvo-Arredondo, J., Riego-Ruiz, L., Castaño, I., et al. 2008. High resistance to oxidative stress in the fungal pathogen Candida glabrata is mediated by a single catalase, Cta1p, and is controlled by the transcription factors Yap1p, Skn7p, Msn2p, and Msn4p. Eukaryotic Cell. 7(5): 814-825.

da Silva, A., Lee, K.K., Raziunaite, I., Schaefer, K., Wagener, J., Yadav, B., et al. 2016. Cell biology of Candida albicans-host interactions. Current Opinion in Microbiology. 34: 111-118.

Dujon, B., Sherman, D., Fischer, G., Durrens, P., Casaregola, S. y Lafontaine, I. 2004. Genome evolution in yeasts. Nature. 430: 35-44.

Eggimann, P., Garbino, J.y Pittet, D. 2003. Epidemiology of Candida species infections in critically ill non-immunosuppressed patients. The Lancet Infectious Diseases. 3(11): 685-702.

Franzot, S.P., Mukherjee, J., Cherniak, R., Chen, L.C., Hamdan, J.S. y Casadevall, A. 1998. Microevolution of a estándar strain of Cryptococcus neoformans resulting in differences in virulence and other phenotypes. Infection and Immunity. 66(1): 89-97.

Freisleben, S.H. y Jäger, A.K. 2014. Correlation between plant secondary metabolites and their antifungal mechanisms-a review. Medicinal and Aromatic Plants. 3(2): 1-6.

García-López, J.C., Álvarez-Fuentes, G., Pinos-Rodríguez, J.M., Jasso-Pineda, Y., Contreras-Treviño, H. I., CamachoEscobar, M. A. et al. 2017. Anti-inflammatory Effects of Chrysactinia mexicana Gray Extract in Growing Chicks (Gallus gallusdomesticus) Challenged with LPS and PHA. International Journal of Current Microbiology and Applied Sciences. 6(1): 550-562.

Guinea, J. 2014. Global trends in the distribution of Candida species causing candidemia. Clinical Microbiology and Infection. 20: 5-10. 
Hoehamer, C.F., Cummings, E.D., Hilliard, G.M. y Rogers, P.D. 2010. Changes in the proteome of Candida albicans in response to azole, polyene, and echinocandin antifungal agents. Antimicrobial Agents and Chemotherapy. 54(5): 1655-1664.

Hoet, S., Stevigny, C., Hérent, M.F. y Quetin-Leclercq, J. 2006. Antitrypanosomal compounds from the leaf essential oil of Strychnos spinosa. Planta Medica. 72(5): 480-482.

Horn, D.L., Neofytos, D., Anaissie, E.J., Fishman, J.A., Steinbach, W.J., Olyaei, A.J., et al. 2009. Epidemiology and outcomes of candidemia in 2019 patients: data from the prospective antifungal therapy alliance registry. Clinical Infectious Diseases. 48(12): 1695-1703.

Hyldgaard, M., Mygind, T. y Meyer, R.L. 2012. Essential oils in food preservation: mode of action, synergies, and interactions with food matrix components. Frontiers in Microbiology. 3: 12.

Ipek, E., Zeytinoglu, H., Okay, S., Tuylu, B.A., Kurkcuoglu, M. y Baser, K.H.C. 2005. Genotoxicity and antigenotoxicity of Origanum oil and carvacrol evaluated by Ames Salmonella/ microsomal test. Food Chemistry. 93(3): 551-556.

Jakubowski, W., Biliński, T. y Bartosz, G. 2000. Oxidative stress during aging of stationary cultures of the yeast Saccharomyces cerevisiae. Free Radical Biology and Medicine. 28(5): 659-664.

Jiang, C., Dong, D., Yu, B., Cai, G., Wang, X., Ji, Y., et al. 2013. Mechanisms of azole resistance in 52 clinical isolates of Candida tropicalis in China. Journal of Antimicrobial Chemotherapy. 68(4): 778-785.

Köler, J.R., Casadevall, A. y Perfect, J. 2015. The spectrum of fungi that infects humans. Cold Spring Harbor Perspectives in Medicine. 5(1): a019273.

López-Fuentes, E., Gutiérrez-Escobedo, G., Timmermans, B., Van Dijck, P., De Las Peñas, A. y Castaño, I. 2018. Candida glabrata's genome plasticity confers a unique pattern of expressed cell wall proteins. Journal of Fungi. 4(2): 67.

Magalhães, Y.C., Bomfim, M.R.Q., Melônio, L.C., Ribeiro, P., Cosme, L.M., Rhoden, C.R., et al. 2015. Clinical significance of the isolation of Candida species from hospitalized patients. Brazilian Journal of Microbiology. 46(1): 117-123.

Mahilrajan, S., Nandakumar, J., Kailayalingam, R., Manoharan, N.A. y SriVijeindran, S. 2014. Screening the antifungal activity of essential oils against decay fungi from palmyrah leaf handicrafts. Biological Research. 47(1): 35.

Moran, C., Grussemeyer, C.A., Spalding, J.R., Benjamin, D.K. y Reed, S.D. 2010. Comparison of costs, length of stay, and mortality associated with Candida glabrata and Candida albicans bloodstream infections. American Journal of Infection Control. 38(1): 78-80.

Nazzaro, F., Fratianni, F., De Martino, L., Coppola, R. y De Feo, V. 2013. Effect of essential oils on pathogenic bacteria. Pharmaceuticals. 6(12): 1451-1474.

Orkwis, B.R., Davies, D.L., Kerwin, C.L., Sanglard, D. y Wykoff, D.D. 2010. Novel acid phosphatase in Candida glabrata suggests selective pressure and niche specialization in the phosphate signal transduction pathway. Genetics. 186(3): 885-895.

Pineda-Díaz, J., Gómez-Meraz, Y., Xoconostle-Cázares, B. y GarcíaMena, J. 2017. Detección de Candida glabrata en mujeres mexicanas sanas y con candidiasis vulvovaginal recurrente. Ginecología y Obstetricia de México. 85(2): 71-79.

Poláková, S., Blume, C., Zárate, J.A., Mentel, M., Jørck-Ramberg, D., Stenderup, J., et al. 2009. Formation of new chromosomes as a virulence mechanism in yeast Candida glabrata. Proceedings of the National Academy of Sciences. 106(8): 2688-2693.

Roche, C.M., Blanch, H.W., Clark, D.S. y Glass, N.L. 2013. Physiological role of acyl coenzyme A synthetase homologs in lipid metabolism in Neurospora crassa. Eukaryotic Cell. 12(9): 1244-1257.

Santana-Rios, G., Orner, G.A., Amantana, A., Provost, C., Wu, S.-Y.y Dashwood, R.H. 2001. Potent antimutagenic activity of white tea in comparison with green tea in the Salmonella assay. Mutation Research/Genetic Toxicology and Environmental Mutagenesis. 495(1): 61-74.

Santos, S.B.D., Sabadin, C.E.S., Mario, D.N., Rigo, L. y Barbosa, D.A. 2018. Presence of Candida spp. and candidiasis in liver transplant patients. Anais Brasileiros de Dermatologia. 93(3): 356-361.

Secretaria de Salud. 2015. Informe Anual 2015: Red Hospitalaria de Vigilancia Epidemiológica. Recuperado de https://www. gob.mx/cms/uploads/attachment/file/212974/infoanual rhove 2015.pdf México. (Accesado 04/06/2020).

Selmecki, A., Forche, A. y Berman, J. 2010. Genomic plasticity of the human fungal pathogen Candida albicans. Eukaryotic Cell. 9(7): 991-1008.

Underhill, D.M. y lliev, I.D. 2014. The mycobiota: interactions between commensal fungi and the host immune system. Nature Reviews Immunology. 14(6): 405-416.

Zore, G.B., Thakre, A.D., Jadhav, S. y Karuppayil, S. M. 2011. Terpenoids inhibit Candida albicans growth by affecting membrane integrity and arrest of cell cycle. Phytomedicine. 18(13): 1181-1190. 